\title{
Article \\ New Fractional Dynamic Inequalities via Conformable Delta Derivative on Arbitrary Time Scales
}

\author{
Ahmed A. El-Deeb ${ }^{1, *}$, Hijaz Ahmad ${ }^{2}$ (D) and Jan Awrejcewicz ${ }^{3, *}$ (D) \\ 1 Department of Mathematics, Faculty of Science, Al-Azhar University, Nasr City 11884, Cairo, Egypt \\ 2 Section of Mathematics, International Telematic University Uninettuno, Corso Vittorio Emanuele II, 39, \\ 00186 Rome, Italy; hijaz555@gmail.com \\ 3 Department of Automation, Biomechanics and Mechatronics, Lodz University of Technology, 1/15 \\ Stefanowski St., 90-924 Lodz, Poland \\ * Correspondence: ahmedeldeeb@azhar.edu.eg (A.A.E.-D.); jan.awrejcewicz@p.lodz.pl (J.A.); \\ Tel.: +20-1098802022 (A.A.E.-D.)
}

check for updates

Citation: El-Deeb, A.A.; Ahmed, H.; Awrejcewicz, J. New Fractional

Dynamic Inequalities via

Conformable Delta Derivative on Arbitrary Time Scales. Symmetry 2021, 13, 2049. https://doi.org/10.3390/ sym 13112049

Academic Editor: Ioan Rașa

Received: 4 October 2021

Accepted: 25 October 2021

Published: 31 October 2021

Publisher's Note: MDPI stays neutral with regard to jurisdictional claims in published maps and institutional affiliations.

\section{Copyright: (c) 2021 by the authors.} Licensee MDPI, Basel, Switzerland. This article is an open access article distributed under the terms and conditions of the Creative Commons Attribution (CC BY) license (https:// creativecommons.org/licenses/by/ $4.0 /)$.

\begin{abstract}
Building on the work of Josip Pečarić in 2013 and 1982 and on the work of Srivastava in 2017. We prove some new $\alpha$-conformable dynamic inequalities of Steffensen-type on time scales. In the case when $\alpha=1$, we obtain some well-known time scale inequalities due to Steffensen inequalities. For some specific time scales, we further show some relevant inequalities as special cases: $\alpha$-conformable integral inequalities and $\alpha$-conformable discrete inequalities. Symmetry plays an essential role in determining the correct methods to solve dynamic inequalities.
\end{abstract}

Keywords: Steffensen's inequality; dynamic inequality; $\alpha$-conformable calculus; time scale

\section{Introduction}

Every nonempty arbitrary closed subset of the real numbers is called time-scale $\mathbb{T}$. We suppose that $\mathbb{T}$ has a standard topology on real numbers $\mathbb{R}$. More details about the definitions and concepts of time-scales calculus and $\alpha$-conformable calculus can be found in [1-18]. We suppose that $\sigma: \mathbb{T} \rightarrow \mathbb{T}$, the forward jump operator, by

$$
\sigma(t):=\inf \{s \in \mathbb{T}: s>t\}, \quad t \in \mathbb{T}
$$

and that $\rho: \mathbb{T}: \rightarrow \mathbb{T}$, the backward jump operator, by

$$
\rho(t):=\sup \{s \in \mathbb{T}: s<t\}, \quad t \in \mathbb{T} .
$$

In (1) and (2), we set sup $\mathbb{T}=\inf \varnothing$ (i.e., $\sigma(t)=t$ if $t$ is the minimum of $\mathbb{T}$ ) and inf $\mathbb{T}=\sup \varnothing$ (i.e., $\rho(t)=t$ if $t$ is the maximum), where $\varnothing$ is the empty set.

Definition 1. Let $\eta: \mathbb{T} \rightarrow \mathbb{R}, t \in \mathbb{T}^{k}$, and $\alpha \in(0,1]$. For $t>0$, we define $T_{\alpha}^{\Delta}(\eta)(t)$ as the number (provided it exists) with the property that, given any $\epsilon>0$, there is a $\delta$ neighbrhood $U_{t} \subset \mathbb{T}$ of $t, \delta>0$, such that

$$
\left|[\eta(\sigma(t))-\eta(s)] t^{1-\alpha}-T_{\alpha}^{\Delta}(\eta)(t)[\sigma(t)-s]\right| \leq \varepsilon|\sigma(t)-s|
$$

for all $s \in U_{t}$. We call $T_{\alpha}^{\Delta}(\eta)(t)$ the conformable fractional derivative of $\eta$ of order $\alpha$ at $t$, and we define a conformable fractional derivative on $\mathbb{T}$ at 0 , as $T_{\alpha}^{\Delta}(\eta)(0)=\lim _{t \rightarrow 0+} T_{\alpha}^{\Delta}(\eta)(t)$.

For any time-scales $\mathbb{T}$, we have

$$
\begin{aligned}
(\eta)^{\Delta_{\alpha}}(t) & =(\eta)^{\Delta}(t) t^{1-\alpha} \\
\int_{a}^{b} \eta(t) \Delta_{\alpha} t & =\int_{a}^{b} \eta(t) t^{\alpha-1} \Delta t
\end{aligned}
$$


Recently, a massive range of dynamic inequalities on time scales has been investigated by using exclusive authors who have been inspired with the aid of a few applications (see [19-32]). Symmetry plays an essential role in determining the correct methods to solve dynamic inequalities. Some authors created different results regarding fractional calculus on time scales to provide associated dynamic inequalities (see [33-36]).

In [37], Pečarić introduced the following result.

Theorem 1. Let $\hat{\lambda}, \hat{\lambda_{1}}, \hat{\lambda_{2}}:\left[\ell_{1}, \ell_{2}\right] \rightarrow \mathbb{R}$ be integrable functions on $\left[\ell_{1}, \ell_{2}\right]$ such that $\hat{\lambda} / \hat{\lambda_{2}}$ is nonincreasing and $\hat{\lambda_{2}}$ is nonnegative. Furthermore, let $0 \leq \hat{\lambda_{1}}(j) \leq 1 \forall j \in\left[\ell_{1}, \ell_{2}\right]$. Then,

$$
\int_{\ell_{2}-\hat{\Im}}^{\ell_{2}} \hat{\lambda}(\jmath) d \jmath \leq \int_{\ell_{1}}^{\ell_{2}} \hat{\lambda}(\jmath) \hat{\lambda_{1}}(\jmath) d \jmath
$$

where $\hat{\Im}$ gives us the solution of

$$
\int_{\ell_{2}-\hat{\Im}}^{\ell_{2}} \hat{\lambda_{2}}(\jmath) d \jmath=\int_{\ell_{1}}^{\ell_{2}} \hat{\lambda_{2}}(\jmath) \hat{\lambda_{1}}(\jmath) d \jmath
$$

We obtain the reverse of $(4)$, if $\hat{f(j)} / h \hat{(j)}$ is nondecreasing.

Several inequalities such as Hardy's inequality [38,39], Hermite-Hadamard's inequality [40-42], Opial's inequality [43,44], and Steffensen's inequality [45] have been introduced. For example, in 2016, Anderson [46] gave an $\alpha$-conformable version of Steffensen inequality as follows:

Theorem 2. [46] [Fractional Steffensen's inequality] Suppose $\alpha \in(0,1]$ and $\ell_{1}, \ell_{2} \in \mathbb{R}$ such that $0 \leq \ell_{1} \leq \ell_{2}$. Suppose that $\Pi:\left[\ell_{1}, \ell_{2}\right] \rightarrow[0, \infty)$ and $\Gamma:\left[\ell_{1}, \ell_{2}\right] \rightarrow[0,1]$ are $\alpha$-fractional integrable functions on $\left[\ell_{1}, \ell_{2}\right]$ with $\omega$ decreasing. We have

$$
\int_{\ell_{2}-\mho}^{\ell_{2}} \omega(\jmath) d_{\alpha \jmath} \leq \int_{\ell_{1}}^{\ell_{2}} \omega(\jmath) \Gamma(\jmath) d_{\alpha \jmath} \leq \int_{\ell_{1}}^{\ell_{1}+\mho} \omega(\jmath) d_{\alpha \jmath},
$$

where $\mho=\frac{\alpha\left(\ell_{2}-\ell_{1}\right)}{\ell_{2}^{\alpha}-\ell_{1}^{\alpha}} \int_{\ell_{1}}^{\ell_{2}} \Gamma(\jmath) d_{\alpha \jmath} \in\left[0, \ell_{2}-\ell_{1}\right]$.

In 2017, Sarikaya et al. [45] gave a generalization for Theorem 2 as follows:

Theorem 3. Suppose that $\alpha \in(0,1]$ and $\ell_{1}, \ell_{2} \in \mathbb{R}$ such that $0 \leq \ell_{1} \leq \ell_{2}$. Suppose that $\prod, \Gamma, \Xi:\left[\ell_{1}, \ell_{2}\right] \rightarrow[0, \infty)$ are $\alpha$-fractional integrable functions on $\left[\ell_{1}, \ell_{2}\right]$ with $\omega$ decreasing and $0 \leq \Gamma \leq \Xi$. We have

$$
\int_{\ell_{2}-\mho}^{\ell_{2}} \Xi(\jmath) \omega(\jmath) d_{\alpha \jmath} \leq \int_{\ell_{1}}^{\ell_{2}} \omega(\jmath) \Gamma(\jmath) d_{\alpha \jmath} \leq \int_{\ell_{1}}^{\ell_{1}+\mho} \Xi(\jmath) \omega(\jmath) d_{\alpha \jmath},
$$

where $\mho=\frac{\left(\ell_{2}-\ell_{1}\right)}{\int_{\ell_{1}}^{\ell_{2}} \Xi(j) d_{\alpha \jmath}} \int_{\ell_{1}}^{\ell_{2}} \Gamma(j) d_{\alpha \jmath} \in\left[0, \ell_{2}-\ell_{1}\right]$.

In this article, we explore new generalizations of the integral Steffensen inequality given in $[37,47,48]$ via a conformable integral on a general time-scale measure space. We also retrieve some of the integral inequalities known in the literature as special cases of our tests.

\section{Main Results}

Next, we enroll the accompanying suppositions for the verifications of our primary outcomes: 
( $\left.\Re_{1}\right)\left(\left[\ell_{1}, \ell_{2}\right]_{\mathbb{T}}, \mathfrak{B}\left(\left[\ell_{1}, \ell_{2}\right]_{\mathbb{T}}\right), \hat{\mu}\right)$ is a time-scale measure space with a positive $\sigma$-finite measure on $\mathfrak{B}\left(\left[\ell_{1}, \ell_{2}\right]_{\mathbb{T}}\right)$.

$\left.\left(\Re_{2}\right)\right], \Phi, \digamma:\left[\ell_{1}, \ell_{2}\right]_{\mathbb{T}} \rightarrow \mathbb{R}$ is $\Delta_{\alpha}$-integrable functions on $\left[\ell_{1}, \ell_{2}\right]_{\mathbb{T}}$.

$\left(\Re_{3}\right) I / \digamma$ is nonincreasing, and $\digamma$ is nonnegative.

$\left(\Re_{4}\right) 0 \leq \Phi(\jmath) \leq 1$ for all $\jmath \in\left[\ell_{1}, \ell_{2}\right]_{\mathbb{T}}$.

$\left(\Re_{5}\right) \hat{\Im}$ is a real number.

$\left(\Re_{6}\right)$ I is nonincreasing.

$\left(\Re_{7}\right) 1 \leq \Phi(\jmath) \leq \digamma(\jmath)$ for all $\jmath \in\left[\ell_{1}, \ell_{2}\right]_{\mathbb{T}}$.

( $\left.\Re_{8}\right) 0 \leq \psi(\jmath) \leq \Phi(\jmath) \leq \digamma(\jmath)-\psi(\jmath)$ for all $\jmath \in\left[\ell_{1}, \ell_{2}\right]_{\mathbb{T}}$.

( $\left.\Re_{9}\right) 0 \leq M \leq \Phi(\jmath) \leq 1-M$ for all $\jmath \in\left[\ell_{1}, \ell_{2}\right]_{\mathbb{T}}$.

$\left(\Re_{10}\right) 0 \leq \psi(\jmath) \leq \Phi(\jmath) \leq 1-\psi(\jmath)$ for all $\jmath \in\left[\ell_{1}, \ell_{2}\right]_{\mathbb{T}}$.

$\hat{\Im}$ is the solution of the equations listed below:

( $\left.\Re_{11}\right) \int_{\left[\ell_{1}, \ell_{1}+\Im\right]_{\mathbb{T}}} \digamma(\jmath) \Delta_{\alpha \jmath}=\int_{\left[\ell_{1}, \ell_{2}\right]_{\mathbb{T}}} \digamma(\jmath) \Phi(\jmath) \Delta_{\alpha}$.

( $\left.\left.\left.\Re_{12}\right) \int_{\left[\ell_{2}-\hat{\Im}_{,}, \ell_{2}\right]_{\mathbb{T}}} \digamma(\jmath) \Delta_{\alpha}\right]=\int_{\left[\ell_{1}, \ell_{2}\right]_{\mathbb{T}}} \digamma(\jmath) \Phi(\jmath) \Delta_{\alpha}\right]$.

( $\left.\left.\left.\left.\Re_{13}\right) \int_{\left[\ell_{1}, \ell_{1}+\hat{\Im}\right]_{\mathbb{T}}} \digamma(\jmath) \Delta_{\alpha}\right]=\int_{\left[\ell_{1}, \ell_{2}\right]_{\mathbb{T}}} \Phi(\jmath) \Delta_{\alpha}\right]=\int_{\left[\ell_{2}-\hat{\Im}_{,}, \ell_{2}\right]_{\mathbb{T}}} \digamma(\jmath) \Delta_{\alpha}\right]$.

( $\left.\left.\left.\Re_{14}\right) \int_{\left[\ell_{1}, \ell_{1}+\hat{\Im}\right]_{\mathbb{T}}} \digamma(\jmath) \Delta_{\alpha}\right]=\int_{\left[\ell_{1}, \ell_{2}\right]_{\mathbb{T}}} \Phi(\jmath) \Delta_{\alpha}\right]$.

( $\left.\left.\left.\Re_{15}\right) \int_{\left[\ell_{2}-\hat{\Im}_{,} \ell_{2}\right]_{\mathbb{T}}} \digamma(\jmath) \Delta_{\alpha}\right]=\int_{\left[\ell_{1}, \ell_{2}\right]_{\mathbb{T}}} \Phi(\jmath) \Delta_{\alpha}\right]$.

Now, we are ready to state and prove our main results.

Theorem 4. Let $\Re_{1}, \Re_{2}, \Re_{3}, \Re_{4}$, and $\Re_{11}$ be satisfied. Then,

$$
\int_{\left[\ell_{1}, \ell_{2}\right]_{\mathbb{T}}} I(\jmath) \Phi(\jmath) \Delta_{\alpha} \leq \int_{\left[\ell_{1}, \ell_{1}+\hat{\Im}\right]_{\mathbb{T}}} I(\jmath) \Delta_{\alpha} .
$$

We obtain the reverse of (3) if $I / \digamma$ is nondecreasing.

\section{Proof.}

$$
\begin{aligned}
& \int_{\left[\ell_{1}, \ell_{1}+\hat{\Im}\right]_{\mathbb{T}}} I(j) \Delta_{\alpha \jmath}-\int_{\left[\ell_{1}, \ell_{2}\right]_{\mathbb{T}}} I(j) \Phi(j) \Delta_{\alpha \jmath}
\end{aligned}
$$

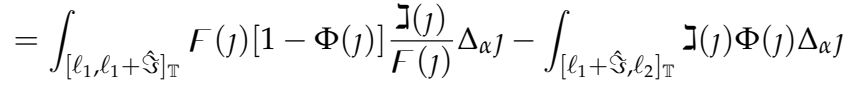

$$
\begin{aligned}
& \left.\left.\geq \frac{I\left(\ell_{1}+\hat{\Im}\right)}{\digamma\left(\ell_{1}+\hat{\Im}\right)} \int_{\left[\ell_{1}, \ell_{1}+\hat{\Im}\right]_{\mathbb{T}}} \digamma(\jmath)[1-\Phi(\jmath)] \Delta_{\alpha}\right]-\int_{\left[\ell_{1}+\hat{\Im}, \ell_{2}\right]_{\mathbb{T}}} I(\jmath) \Phi(\jmath) \Delta_{\alpha}\right] \\
& \left.\left.=\frac{I\left(\ell_{1}+\hat{\Im}\right)}{\digamma\left(\ell_{1}+\hat{\Im}\right)}\left[\int_{\left[\ell_{1}, \ell_{1}+\hat{\Im}\right]_{\mathbb{T}}} \digamma(\jmath) \Delta_{\alpha}\right]-\int_{\left[\ell_{1}, \ell_{1}+\hat{\Im}\right]_{\mathbb{T}}} \digamma(\jmath) \Phi(\jmath) \Delta_{\alpha \jmath}\right]-\int_{\left[\ell_{1}+\hat{\Im}, \ell_{2}\right]_{\mathbb{T}}} I(\jmath) \Phi(\jmath) \Delta_{\alpha}\right] \\
& \left.=\frac{I\left(\ell_{1}+\hat{\Im}\right)}{\digamma\left(\ell_{1}+\hat{\Im}\right)}\left[\int_{\left[\ell_{1}, \ell_{2}\right]_{\mathbb{T}}} \digamma(\jmath) \Phi(j) \Delta_{\alpha} \jmath-\int_{\left[\ell_{1}, \ell_{1}+\hat{\Im}\right]_{\mathbb{T}}} \digamma(\jmath) \Phi(j) \Delta_{\alpha}\right]\right]-\int_{\left[\ell_{1}+\hat{\Im}, \ell_{2}\right]_{\mathbb{T}}} I(j) \Phi(j) \Delta_{\alpha} \jmath \\
& =\frac{J\left(\ell_{1}+\hat{\Im}\right)}{\digamma\left(\ell_{1}+\hat{\Im}\right)} \int_{\left[\ell_{1}+\hat{\Im}, \ell_{2}\right]_{\mathbb{T}}} \digamma(\jmath) \Phi(\jmath) \Delta_{\alpha} \jmath-\int_{\left[\ell_{1}+\hat{\Im}, \ell_{2}\right]_{\mathbb{T}}} I(j) \Phi(\jmath) \Delta_{\alpha} \jmath \\
& =\int_{\left[\ell_{1}+\hat{\Im}, \ell_{2}\right]_{\mathbb{T}}} \digamma(j) \Phi(j)\left(\frac{J\left(\ell_{1}+\hat{\Im}\right)}{\digamma\left(\ell_{1}+\hat{\Im}\right)}-\frac{J(\jmath)}{\digamma(\jmath)}\right) \Delta_{\alpha \jmath} \geq 0 .
\end{aligned}
$$

The proof is complete.

Corollary 1. Putting $\mathbb{T}=\mathbb{R}$ in Theorem 4 , we obtain

$$
\left.\int_{\left[\ell_{1}, \ell_{2}\right]} I(\jmath) \Phi(\jmath) d_{\alpha \jmath} \leq \int_{\left[\ell_{1}, \ell_{1}+\hat{\Im}\right]} I(\jmath) d_{\alpha}\right] .
$$


Remark 1. In the case of $\alpha=1$ in Corollary 1, we recollect [37] [Theorem 1].

Theorem 5. Assumptions $\Re_{1}, \Re_{2}, \Re_{3}, \Re_{4}$, and $\Re_{12}$ imply

$$
\left.\left.\int_{\left[\ell_{2}-\hat{\Im}, \ell_{2}\right]_{\mathbb{T}}} I(j) \Delta_{\alpha}\right] \leq \int_{\left[\ell_{1}, \ell_{2}\right]_{\mathbb{T}}} I(j) \Phi(j) \Delta_{\alpha}\right] .
$$

We obtain the reverse of (4) if $I / \digamma$ is nondecreasing.

\section{Proof.}

$$
\begin{aligned}
& \int_{\left[\ell_{2}-\hat{\xi}, \ell_{2}\right]_{\mathbb{T}}} I(j) \Delta_{\alpha \jmath}-\int_{\left[\ell_{1}, \ell_{2}\right]_{\mathbb{T}}} J(j) \Phi(j) \Delta_{\alpha \jmath}
\end{aligned}
$$

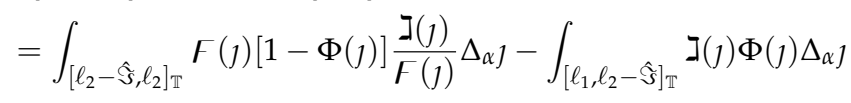

$$
\begin{aligned}
& \left.\left.\leq \frac{J\left(\ell_{2}-\hat{\Im}\right)}{F\left(\ell_{2}-\hat{\Im}\right)} \int_{\left[\ell_{2}-\hat{\Im}, \ell_{2}\right]_{\mathbb{T}}} F(j)[1-\Phi(j)] \Delta_{\alpha}\right]-\int_{\left[\ell_{1}, \ell_{2}-\hat{\Im}\right]_{\mathbb{T}}} I(j) \Phi(j) \Delta_{\alpha}\right] \\
& \left.=\frac{J\left(\ell_{2}-\hat{\Im}\right)}{\digamma\left(\ell_{2}-\hat{\Im}\right)}\left[\int_{\left[\ell_{2}-\hat{\Im}, \ell_{2}\right]_{\mathbb{T}}} \digamma(\jmath) \Delta_{\alpha \jmath}-\int_{\left[\ell_{2}-\hat{\Im}, \ell_{2}\right]_{\mathbb{T}}} \digamma(\jmath) \Phi(\jmath) \Delta_{\alpha \jmath}\right]-\int_{\left[\ell_{1}, \ell_{2}-\hat{\Im}\right]_{\mathbb{T}}} I(\jmath) \Phi(j) \Delta_{\alpha}\right] \\
& \left.\left.=\frac{J\left(\ell_{2}-\hat{\Im}\right)}{F\left(\ell_{2}-\hat{\Im}\right)}\left[\int_{\left[\ell_{1}, \ell_{2}\right]_{\mathrm{T}}} \digamma(j) \Phi(j) \Delta_{\alpha}\right]-\int_{\left[\ell_{2}-\hat{\Im}, \ell_{2}\right]_{\mathrm{T}}} \digamma(\jmath) \Phi(j) \Delta_{\alpha \jmath}\right]-\int_{\left[\ell_{1}, \ell_{2}-\widehat{\Im}\right]_{\mathrm{T}}} J(j) \Phi(j) \Delta_{\alpha}\right] \\
& \left.=\frac{J\left(\ell_{2}-\hat{\Im}\right)}{F\left(\ell_{2}-\hat{\Im}\right)} \int_{\left[\ell_{1}, \ell_{2}-\hat{\Im}\right]_{\mathbb{T}}} F(\jmath) \Phi(j) \Delta_{\alpha}\right]-\int_{\left[\ell_{1}, \ell_{2}-\hat{\Im}\right]_{\mathbb{T}}} I(j) \Phi(j) \Delta_{\alpha} \\
& =\int_{\left[\ell_{1}, \ell_{2}-\hat{\Im}\right]_{\mathbb{T}}} \digamma(j) \Phi(j)\left(\frac{J\left(\ell_{2}-\hat{\Im}\right)}{\digamma\left(\ell_{2}-\hat{\Im}\right)}-\frac{J(j)}{\digamma(j)}\right) \Delta_{\alpha \jmath} \leq 0 .
\end{aligned}
$$

Corollary 2. Putting $\mathbb{T}=\mathbb{R}$ in Theorem 5 ,

$$
\int_{\left[\ell_{2}-\widehat{\Im}, \ell_{2}\right]} I(\jmath) d_{\alpha \jmath} \leq \int_{\left[\ell_{1}, \ell_{2}\right]} I(\jmath) \Phi(\jmath) d_{\alpha]} .
$$

Remark 2. In Corollary 2 and $\alpha=1$, we recapture [37] [Theorem 2].

We will need the following lemma to prove the subsequent results.

Lemma 1. Let $\Re_{1}, \Re_{2}$, and $\Re_{5}$ hold such that

$$
\left.\int_{\left[\ell_{1}, \ell_{1}+\hat{\Im}\right]_{\mathbb{T}}} \digamma(j) \Delta_{\alpha \jmath}=\int_{\left[\ell_{1}, \ell_{2}\right]_{\mathbb{T}}} \Phi(j) \Delta_{\alpha \jmath}=\int_{\left[\ell_{2}-\hat{\Im}, \ell_{2}\right]_{\mathbb{T}}} \digamma(j) \Delta_{\alpha}\right] .
$$

Then,

$$
\begin{aligned}
\int_{\left[\ell_{1}, \ell_{2}\right]_{\mathbb{T}}} I(\jmath) \Phi(\jmath) \Delta_{\alpha}= & \left.\int_{\left[\ell_{1}, \ell_{1}+\hat{\Im}\right]_{\mathbb{T}}}\left(I(\jmath) \digamma(\jmath)-\left[I(\jmath)-J\left(\ell_{1}+\hat{\Im}\right)\right][\digamma(\jmath)-\Phi(\jmath)]\right) \Delta_{\alpha}\right] \\
& \left.+\int_{\left[\ell_{1}+\hat{\Im}, \ell_{2}\right]_{\mathbb{T}}}\left[J(\jmath)-J\left(\ell_{1}+\hat{\Im}\right)\right] \Phi(\jmath) \Delta_{\alpha}\right],
\end{aligned}
$$

and

$$
\begin{aligned}
\int_{\left[\ell_{1}, \ell_{2}\right]_{\mathbb{T}}} I(\jmath) \Phi(\jmath) \Delta_{\alpha} \jmath= & \int_{\left[\ell_{1}, \ell_{2}-\hat{\Im}\right]_{\mathbb{T}}}\left[J(\jmath)-J\left(\ell_{2}-\hat{\Im}\right)\right] \Phi(\jmath) \Delta_{\alpha \jmath} \\
& \left.+\int_{\left[\ell_{2}-\hat{\Im}, \ell_{2}\right]_{\mathbb{T}}}\left(J(\jmath) \digamma(\jmath)-\left[J(\jmath)-J\left(\ell_{2}-\hat{\Im}\right)\right][\digamma(\jmath)-\Phi(\jmath)]\right) \Delta_{\alpha}\right] .
\end{aligned}
$$

Proof. The suppositions of the Lemma imply that

$$
\ell_{1} \leq \ell_{1}+\hat{\Im} \leq \ell_{2} \quad \text { and } \quad \ell_{1} \leq \ell_{2}-\hat{\Im} \leq \ell_{2} \text {. }
$$


Now, we prove (5), and we see that

$$
\begin{aligned}
& \left.\int_{\left[\ell_{1}, \ell_{1}+\hat{\Im}\right]_{\mathbb{T}}}\left(J(\jmath) \digamma(\jmath)-\left[J(\jmath)-J\left(\ell_{1}+\hat{\Im}\right)\right][\digamma(\jmath)-\Phi(\jmath)]\right) \Delta_{\alpha \jmath}-\int_{\left[\ell_{1}, \ell_{2}\right]_{\mathbb{T}}} J(\jmath) \Phi(j) \Delta_{\alpha}\right] \\
& \left.=\int_{\left[\ell_{1}, \ell_{1}+\hat{\Im}\right]_{\mathbb{T}}}\left(J(\jmath) F(\jmath)-J(\jmath) \Phi(\jmath)-\left[I(\jmath)-J\left(\ell_{1}+\hat{\Im}\right)\right][F(\jmath)-\Phi(\jmath)]\right) \Delta_{\alpha}\right] \\
& +\int_{\left[\ell_{1}, \ell_{1}+\hat{\Im}\right]_{\mathbb{T}}} I(j) \Phi(j) \Delta_{\alpha \jmath}-\int_{\left[\ell_{1}, \ell_{2}\right]_{\mathbb{T}}} I(j) \Phi(j) \Delta_{\alpha \jmath} \\
& =\int_{\left[\ell_{1}, \ell_{1}+\hat{\Im}\right]_{\mathbb{T}}} I\left(\ell_{1}+\hat{\Im}\right)[\digamma(\jmath)-\Phi(\jmath)] \Delta_{\alpha \jmath}-\int_{\left[\ell_{1}+\hat{\Im}, \ell_{2}\right]_{\mathbb{T}}} J(\jmath) \Phi(\jmath) \Delta_{\alpha} \jmath \\
& \left.=J\left(\ell_{1}+\hat{\Im}\right)\left(\int_{\left[\ell_{1}, \ell_{1}+\hat{\Im}\right]_{\mathbb{T}}} F(\jmath) \Delta_{\alpha}\right]-\int_{\left[\ell_{1}, \ell_{1}+\hat{\Im}\right]_{\mathbb{T}}} \Phi(j) \Delta_{\alpha \jmath}\right)-\int_{\left[\ell_{1}+\hat{\Im}, \ell_{2}\right]_{\mathbb{T}}} I(\jmath) \Phi(\jmath) \Delta_{\alpha \jmath} .
\end{aligned}
$$

Since

$$
\left.\left.\int_{\left[\ell_{1}, \ell_{1}+\hat{\Im}\right]_{\mathbb{T}}} \digamma(j) \Delta_{\alpha}\right]=\int_{\left[\ell_{1}, \ell_{2}\right]_{\mathbb{T}}} \Phi(j) \Delta_{\alpha}\right]
$$

we have

$$
\begin{aligned}
& J\left(\ell_{1}+\hat{\Im}\right)\left(\int_{\left[\ell_{1}, \ell_{1}+\hat{\Im}\right]_{\mathbb{T}}} \digamma(j) \Delta_{\alpha \jmath}-\int_{\left[\ell_{1}, \ell_{1}+\hat{\Im}\right]_{\mathbb{T}}} \Phi(j) \Delta_{\alpha \jmath}\right)-\int_{\left[\ell_{1}+\hat{\Im}, \ell_{2}\right]_{\mathbb{T}}} I(j) \Phi(j) \Delta_{\alpha \jmath} \\
& \left.=J\left(\ell_{1}+\hat{\Im}\right)\left(\int_{\left[\ell_{1}, \ell_{2}\right]_{\mathbb{T}}} \Phi(\jmath) \Delta_{\alpha}\right]-\int_{\left[\ell_{1}, \ell_{1}+\hat{\Im}\right]_{\mathbb{T}}} \Phi(j) \Delta_{\alpha \jmath}\right)-\int_{\left[\ell_{1}+\hat{\Im}, \ell_{2}\right]_{\mathbb{T}}} I(\jmath) \Phi(\jmath) \Delta_{\alpha \jmath} \\
& \left.=I\left(\ell_{1}+\hat{\Im}\right) \int_{\left[\ell_{1}+\hat{\Im}, \ell_{2}\right]_{\mathbb{T}}} \Phi(\jmath) \Delta_{\alpha \jmath}-\int_{\left[\ell_{1}+\hat{\Im}, \ell_{2}\right]_{\mathbb{T}}} I(\jmath) \Phi(\jmath) \Delta_{\alpha \jmath}\right) \\
& \left.\left.=\int_{\left[\ell_{1}+\hat{\Im}, \ell_{2}\right]_{\mathbb{T}}}[]\left(\ell_{1}+\hat{\Im}\right)-J(\jmath)\right] \Phi(\jmath) \Delta_{\alpha}\right] .
\end{aligned}
$$

A combination of (7) and (8) led to the required integral identity (5) asserted by the Lemma. The integral identity (10) can be proved similarly. The proof is completed.

Corollary 3. Putting $\mathbb{T}=\mathbb{R}$ in Lemma 1, we obtain

$$
\begin{aligned}
\int_{\left[\ell_{1}, \ell_{2}\right]} I(\jmath) \Phi(j) d_{\alpha}= & \left.\int_{\left[\ell_{1}, \ell_{1}+\hat{\Im}\right]}\left(I(j) \digamma(\jmath)-\left[I(\jmath)-J\left(\ell_{1}+\hat{\Im}\right)\right][\digamma(\jmath)-\Phi(\jmath)]\right) d_{\alpha}\right] \\
& +\int_{\left[\ell_{1}+\hat{\Im}, \ell_{2}\right]}\left[J(\jmath)-J\left(\ell_{1}+\hat{\Im}\right)\right] \Phi(\jmath) d_{\alpha \jmath}
\end{aligned}
$$

and

$$
\begin{aligned}
& \int_{\left[\ell_{1}, \ell_{2}\right]} I(\jmath) \Phi(\jmath) d_{\alpha \jmath}=\int_{\left[\ell_{1}, \ell_{2}-\hat{\Im}\right]}\left[I(\jmath)-I\left(\ell_{2}-\hat{\Im}\right)\right] \Phi(\jmath) d_{\alpha \jmath} \\
& \left.+\int_{\left[\ell_{2}-\hat{\Im}, \ell_{2}\right]}\left(\exists(\jmath) \digamma(\jmath)-\left[I(\jmath)-I\left(\ell_{2}-\hat{\Im}\right)\right][\digamma(\jmath)-\Phi(\jmath)]\right) d_{\alpha}\right] .
\end{aligned}
$$

such that

$$
\int_{\left[\ell_{1}, \ell_{1}+\hat{\Im}\right]} \digamma(j) d_{\alpha]}=\int_{\left[\ell_{1}, \ell_{2}\right]} \Phi(j) d_{\alpha]}=\int_{\left[\ell_{2}-\Im, \ell_{2}\right]} \digamma(j) d_{\alpha]} .
$$

Theorem 6. Suppose that $\Re_{1}, \Re_{2}, \Re_{6}, \Re_{7}$, and $\Re_{13}$ give

$$
\begin{aligned}
& \left.\int_{\left[\ell_{2}-\hat{\Im}, \ell_{2}\right]_{\mathbb{T}}} I(\jmath) \digamma(j) \Delta_{\alpha}\right] \leq \int_{\left[\ell_{2}-\hat{\Im}, \ell_{2}\right]_{\mathbb{T}}}\left(I(\jmath) \digamma(\jmath)-\left[I(\jmath)-I\left(\ell_{2}-\hat{\Im}\right)\right][\digamma(\jmath)-\Phi(j)]\right) \Delta_{\alpha} \jmath \\
& \leq \int_{\left[\ell_{1}, \ell_{2}\right]_{\mathbb{T}}} I(\jmath) \Phi(\jmath) \Delta_{\alpha} \jmath \\
& \leq \int_{\left[\ell_{1}, \ell_{1}+\hat{\Im}\right]_{\mathbb{T}}}\left(\exists(\jmath) \digamma(\jmath)-\left[I(\jmath)-\beth\left(\ell_{1}+\hat{\Im}\right)\right][\digamma(\jmath)-\Phi(\jmath)]\right) \Delta_{\alpha} \\
& \left.\leq \int_{\left[\ell_{1}, \ell_{1}+\hat{\Im}\right]_{\mathbb{T}}} I(\jmath) \digamma(j) \Delta_{\alpha}\right] \text {. }
\end{aligned}
$$


Proof. In the perspective of the considerations that the function $I$ is nonincreasing on $\left[\ell_{1}, \ell_{2}\right]$ and $0 \leq \Phi(j) \leq \digamma(j)$ for all $\jmath \in\left[\ell_{1}, \ell_{2}\right]$, we infer that

$$
\int_{\left[\ell_{1}, \ell_{2}-\hat{\Im}\right]_{\mathbb{T}}}\left[I(j)-I\left(\ell_{2}-\hat{\Im}\right)\right] \Phi(j) \Delta_{\alpha} \geq 0,
$$

and

$$
\left.\int_{\left[\ell_{2}-\hat{\Im}, \ell_{2}\right]_{\mathbb{T}}}\left[\exists\left(\ell_{2}-\hat{\Im}\right)-J(j)\right][\digamma(j)-\Phi(j)] \Delta_{\alpha}\right] \geq 0 .
$$

Using (5), (11), and (12), we find that

$$
\begin{aligned}
& \left.\left.\int_{\left[\ell_{2}-\hat{\Im}, \ell_{2}\right]_{\mathbb{T}}} I(\jmath) \digamma(j) \Delta_{\alpha}\right] \leq \int_{\left[\ell_{2}-\hat{\Im}, \ell_{2}\right]_{\mathbb{T}}}\left(I(\jmath) \digamma(\jmath)-\left[I(\jmath)-I\left(\ell_{2}-\hat{\Im}\right)\right][\digamma(\jmath)-\Phi(\jmath)]\right) \Delta_{\alpha}\right] \\
& \left.\leq \int_{\left[\ell_{1}, \ell_{2}\right]_{\mathbb{T}}} J(\jmath) \Phi(\jmath) \Delta_{\alpha}\right] \\
& \left.\int_{\left[\ell_{1}, \ell_{2}\right]_{\mathbb{T}}} I(\jmath) \Phi(\jmath) \Delta_{\alpha}\right] \leq \int_{\left[\ell_{1}, \ell_{1}+\hat{\Im}\right]_{\mathbb{T}}}\left(I(\jmath) \digamma(\jmath)-\left[I(\jmath)-I\left(\ell_{1}+\hat{\Im}\right)\right][\digamma(\jmath)-\Phi(\jmath)]\right) \Delta_{\alpha} \\
& \leq \int_{\left[\ell_{1}, \ell_{1}+\hat{\Im}\right]_{\mathbb{T}}} I(j) \digamma(\jmath) \Delta_{\alpha]},
\end{aligned}
$$

The confirmation is finished by joining the integral inequalities (13) and (14).

Corollary 4. Putting $\mathbb{T}=\mathbb{R}$ in Theorem 6, we have

$$
\begin{aligned}
& \int_{\left[\ell_{2}-\hat{\Im}, \ell_{2}\right]} I(\jmath) \digamma(j) d_{\alpha \jmath} \leq \int_{\left[\ell_{2}-\hat{\Im}, \ell_{2}\right]}\left(I(\jmath) \digamma(j)-\left[I(\jmath)-I\left(\ell_{2}-\hat{\Im}\right)\right][\digamma(\jmath)-\Phi(\jmath)]\right) d_{\alpha \jmath} \\
& \leq \int_{\left[\ell_{1}, \ell_{2}\right]} J(\jmath) \Phi(\jmath) d_{\alpha \jmath} \\
& \leq \int_{\left[\ell_{1}, \ell_{1}+\hat{\Im}\right]}\left(I(\jmath) \digamma(\jmath)-\left[I(\jmath)-I\left(\ell_{1}+\hat{\Im}\right)\right][\digamma(\jmath)-\Phi(\jmath)]\right) d_{\alpha \jmath} \\
& \left.\leq \int_{\left[\ell_{1}, \ell_{1}+\hat{\Im}\right]} \exists(\jmath) \digamma(\jmath) d_{\alpha}\right] \text {. }
\end{aligned}
$$

Remark 3. We can reclaim [48] [Theorem 1] in Corollary 4 by taking $\alpha=1$.

Theorem 7. Assume that $\Re_{1}, \Re_{2}, \Re_{6}, \Re_{8}$, and $\Re_{13}$ are fulfilled. Then,

$$
\begin{aligned}
& \left.\int_{\left[\ell_{2}-\hat{\Im}, \ell_{2}\right]_{\mathbb{T}}} I(\jmath) \digamma(\jmath) \Delta_{\alpha} \jmath+\int_{\left[\ell_{1}, \ell_{2}\right]_{\mathbb{T}}}\left|\left[J(\jmath)-J\left(\ell_{2}-\hat{\Im}\right)\right] \psi(\jmath)\right| \Delta_{\alpha}\right] \\
& \leq \int_{\left[\ell_{1}, \ell_{2}\right]_{\mathbb{T}}} I(\jmath) \Phi(\jmath) \Delta_{\alpha} \jmath \\
& \left.\left.\leq \int_{\left[\ell_{1}, \ell_{1}+\hat{\Im}\right]_{\mathbb{T}}} I(j) \digamma(\jmath) \Delta_{\alpha}\right]-\int_{\left[\ell_{1}, \ell_{2}\right]_{\mathbb{T}}}\left|\left[I(\jmath)-I\left(\ell_{1}+\hat{\Im}\right)\right] \psi(\jmath)\right| \Delta_{\alpha}\right],
\end{aligned}
$$

Proof. Clearly, function $]$ is nonincreasing on $\left[\ell_{1}, \ell_{2}\right]$ and $0 \leq \psi(\jmath) \leq \Phi(\jmath) \leq \digamma(\jmath)-\psi(\jmath)$ for all $\jmath \in\left[\ell_{1}, \ell_{2}\right]$, and we obtain

$$
\begin{aligned}
& \left.\int_{\left[\ell_{1}, \ell_{1}+\hat{\Im}\right]_{\mathbb{T}}}\left[I(\jmath)-J\left(\ell_{1}+\hat{\Im}\right)\right][\digamma(j)-\Phi(\jmath)] \Delta_{\alpha}\right]+\int_{\left[\ell_{1}+\hat{\Im}, \ell_{2}\right]_{\mathbb{T}}}\left[I\left(\ell_{1}+\hat{\Im}\right)-J(\jmath)\right] \Phi(j) \Delta_{\alpha \jmath} \\
& \left.=\int_{\left[\ell_{1}, \ell_{1}+\hat{\Im}\right]_{\mathbb{T}}}\left|J(\jmath)-J\left(\ell_{1}+\hat{\Im}\right)\right|[\digamma(\jmath)-\Phi(\jmath)] \Delta_{\alpha} \jmath+\int_{\left[\ell_{1}+\hat{\Im}, \ell_{2}\right]_{\mathbb{T}}}\left|J\left(\ell_{1}+\hat{\Im}\right)-J(\jmath)\right| \Phi(\jmath) \Delta_{\alpha}\right] \\
& \left.\left.\geq \int_{\left[\ell_{1}, \ell_{1}+\hat{\Im}\right]_{\mathbb{T}}}\left|J(\jmath)-J\left(\ell_{1}+\hat{\Im}\right)\right| \psi(\jmath) \Delta_{\alpha}\right]+\int_{\left[\ell_{1}+\hat{\Im}, \ell_{2}\right]_{\mathbb{T}}}\left|J\left(\ell_{1}+\hat{\Im}\right)-J(\jmath)\right| \psi(\jmath) \Delta_{\alpha}\right] \\
& \left.\geq \int_{\left[\ell_{1}, \ell_{2}\right]_{\mathbb{T}}}\left|\left[I(\jmath)-I\left(\ell_{1}+\hat{\Im}\right)\right] \psi(j)\right| \Delta_{\alpha}\right] .
\end{aligned}
$$


Additionally,

$$
\begin{aligned}
& \left.\int_{\left[\ell_{1}, \ell_{1}+\hat{\Im}\right]_{\mathbb{T}}}\left[I(\jmath)-I\left(\ell_{1}+\hat{\Im}\right)\right][\digamma(\jmath)-\Phi(\jmath)] \Delta_{\alpha} \jmath+\int_{\left[\ell_{1}+\hat{\Im}, \ell_{2}\right]_{\mathbb{T}}}\left[I\left(\ell_{1}+\hat{\Im}\right)-I(\jmath)\right] \Phi(\jmath) \Delta_{\alpha}\right] \\
& \geq \int_{\left[\ell_{1}, \ell_{2}\right]_{\mathbb{T}}}\left|\left[I(\jmath)-J\left(\ell_{1}+\hat{\Im}\right)\right] \psi(\jmath)\right| \Delta_{\alpha} \jmath .
\end{aligned}
$$

Similarly, we find that

$$
\begin{aligned}
& \left.\int_{\left[\ell_{1}, \ell_{2}-\hat{\Im}\right]_{\mathbb{T}}}\left[I(\jmath)-I\left(\ell_{2}-\hat{\Im}\right)\right] \Phi(j) \Delta_{\alpha} \jmath+\int_{\left[\ell_{2}-\hat{\Im}, \ell_{2}\right]_{\mathbb{T}}}\left[I\left(\ell_{2}-\hat{\Im}\right)-I(\jmath)\right][\digamma(\jmath)-\Phi(j)] \Delta_{\alpha}\right] \\
& \geq \int_{\left[\ell_{1}, \ell_{2}\right]_{\mathbb{T}}}\left|\left[I(\jmath)-I\left(\ell_{2}-\hat{\Im}\right)\right] \psi(\jmath)\right| \Delta_{\alpha} J .
\end{aligned}
$$

By combining (5), (10), (16), and (17), we arrive at the inequality (15) asserted by Theorem 7.

Corollary 5. Putting $\mathbb{T}=\mathbb{R}$ in Theorem 7 , we have

$$
\begin{aligned}
& \int_{\left[\ell_{2}-\hat{\Im}, \ell_{2}\right]} I(\jmath) \digamma(\jmath) d_{\alpha \jmath}+\int_{\left[\ell_{1}, \ell_{2}\right]}\left|\left[I(\jmath)-I\left(\ell_{2}-\hat{\Im}\right)\right] \psi(\jmath)\right| d_{\alpha \jmath} \\
& \leq \int_{\left[\ell_{1}, \ell_{2}\right]} I(\jmath) \Phi(\jmath) d_{\alpha \jmath} \\
& \left.\leq \int_{\left[\ell_{1}, \ell_{1}+\hat{\Im}\right]} I(\jmath) \digamma(\jmath) d_{\alpha \jmath}-\int_{\left[\ell_{1}, \ell_{2}\right]}\left|\left[I(\jmath)-I\left(\ell_{1}+\hat{\Im}\right)\right] \psi(\jmath)\right| d_{\alpha}\right] .
\end{aligned}
$$

Remark 4. If we take $\alpha=1$, in Corollary 5, we recapture [48] [Theorem 2].

Theorem 8. Let $\Re_{1}, \Re_{2}, \Re_{6}$, and $\Re_{9}$ be satisfied, and

$$
0 \leq \hat{\Im}_{1} \leq \int_{\left[\ell_{1}, \ell_{2}\right]_{\mathbb{T}}} \Phi(j) \Delta_{\alpha} \leq \hat{\Im}_{2} \leq \ell_{2}-\ell_{1}
$$

Then,

$$
\begin{aligned}
& \int_{\left[\ell_{2}-\hat{\Im}_{1}, \ell_{2}\right]_{\mathbb{T}}} I(\jmath) \Delta_{\alpha} \jmath+I\left(\ell_{2}\right)\left(\int_{\left[\ell_{1}, \ell_{2}\right]_{\mathbb{T}}} \Phi(\jmath) \Delta_{\alpha} \jmath-\hat{\Im}_{1}\right) \\
& +M \int_{\left[\ell_{1}, \ell_{2}\right]_{\mathbb{T}}}\left|\exists(\jmath)-f\left(\ell_{2}-\int_{\left[\ell_{1}, \ell_{2}\right]_{\mathbb{T}}} \Phi(\jmath) \Delta_{\alpha \jmath}\right)\right| \Delta_{\alpha} \jmath \\
& \leq \int_{\left[\ell_{1}, \ell_{2}\right]_{\mathbb{T}}} I(\jmath) \Phi(\jmath) \Delta_{\alpha} \jmath \\
& \leq \int_{\left[\ell_{1}, \ell_{1}+\hat{\Im}_{2}\right]_{\mathbb{T}}} I(\jmath) \Delta_{\alpha} J-I\left(\ell_{2}\right)\left(\hat{\Im}_{2}-\int_{\left[\ell_{1}, \ell_{2}\right]_{\mathbb{T}}} \Phi(\jmath) \Delta_{\alpha \jmath}\right) \\
& -M \int_{\left[\ell_{1}, \ell_{2}\right]_{\mathbb{T}}}\left|I(\jmath)-f\left(\ell_{1}+\int_{\left[\ell_{1}, \ell_{2}\right]_{\mathbb{T}}} \Phi(\jmath) \Delta_{\alpha \jmath}\right)\right| \Delta_{\alpha} \text {. }
\end{aligned}
$$

Proof. By using straightforward calculations, we have

$$
\begin{aligned}
& \int_{\left[\ell_{1}, \ell_{2}\right]_{\mathbb{T}}} I(\jmath) \Phi(\jmath) \Delta_{\alpha} J-\int_{\left[\ell_{1}, \ell_{1}+\hat{\Im}_{2}\right]_{\mathbb{T}}} I(\jmath) \Delta_{\alpha \jmath}+I\left(\ell_{2}\right)\left(\hat{\Im}_{2}-\int_{\left[\ell_{1}, \ell_{2}\right]_{\mathbb{T}}} \Phi(\jmath) \Delta_{\alpha \jmath}\right) \\
& =\int_{\left[\ell_{1}, \ell_{2}\right]_{\mathbb{T}}} I(j) \Phi(j) \Delta_{\alpha} J-\int_{\left[\ell_{1}, \ell_{1}+\hat{\Im}_{2}\right]_{\mathbb{T}}} I(j) \Delta_{\alpha} j+\int_{\left[\ell_{1}, \ell_{1}+\hat{\Im}_{2}\right]_{\mathbb{T}}} I\left(\ell_{2}\right) \Delta_{\alpha}-\int_{\left[\ell_{1}, \ell_{2}\right]_{\mathbb{T}}} I\left(\ell_{2}\right) \Phi(j) \Delta_{\alpha} J \\
& \left.=\int_{\left[\ell_{1}, \ell_{2}\right]_{\mathbb{T}}}\left[I(j)-I\left(\ell_{2}\right)\right] \Phi(j) \Delta_{\alpha} J-\int_{\left[\ell_{1}, \ell_{1}+\hat{\Im}_{2}\right]_{\mathbb{T}}}\left[I(j)-I\left(\ell_{2}\right)\right] \Delta_{\alpha}\right] \\
& \left.\leq \int_{\left[\ell_{1}, \ell_{2}\right]_{\mathbb{T}}}\left[J(\jmath)-J\left(\ell_{2}\right)\right] \Phi(j) \Delta_{\alpha}\right]-\int_{\left[\ell_{1}, \ell_{1}+\int_{\left[\ell_{1}, \ell_{2}\right]_{\mathbb{T}}} \Phi(\jmath) \Delta_{\alpha \jmath}\right]}\left[J(\jmath)-J\left(\ell_{2}\right)\right] \Delta_{\alpha \jmath},
\end{aligned}
$$


where we used the theorem's hypotheses

$$
\left.\ell_{1} \leq \ell_{1}+\hat{\Im}_{1} \leq \ell_{1}+\int_{\left[\ell_{1}, \ell_{2}\right]_{\mathbb{T}}} \Phi(j) \Delta_{\alpha}\right] \leq \ell_{1}+\hat{\Im}_{2} \leq \ell_{2}
$$

and

$$
I(j)-I\left(\ell_{2}\right) \geq 0 \text { for all } \jmath \in\left[\ell_{1}, \ell_{2}\right] .
$$

The function $I(j)-I\left(\ell_{2}\right)$ is nonincreasing and integrable on $\left[\ell_{1}, \ell_{2}\right]$, and by applying Theorem 7 with $\digamma(\jmath)=1, \psi(\jmath)=M$ and $\Xi(\jmath)$ replaced by $\Xi(\jmath)-\Xi\left(\ell_{2}\right)$,

$$
\begin{gathered}
\left.\int_{\left[\ell_{1}, \ell_{2}\right]_{\mathbb{T}}}\left[I(\jmath)-I\left(\ell_{2}\right)\right] \Phi(j) \Delta_{\alpha \jmath}-\int_{\left[\ell_{1}, \ell_{1}+\int_{\left[\ell_{1}, \ell_{2}\right]_{\mathbb{T}}} \Phi(j) \Delta_{\alpha \jmath}\right]}\left[I(\jmath)-I\left(\ell_{2}\right)\right] \Delta_{\alpha}\right] \\
\leq-M \int_{\left[\ell_{1}, \ell_{2}\right]_{\mathbb{T}}}\left|J(\jmath)-f\left(\ell_{1}+\int_{\left[\ell_{1}, \ell_{2}\right]_{\mathbb{T}}} \Phi(\jmath) \Delta_{\alpha \jmath}\right)\right| \Delta_{\alpha} .
\end{gathered}
$$

From (19) and (20), we obtain

$$
\begin{gathered}
\left.\int_{\left[\ell_{1}, \ell_{2}\right]_{\mathbb{T}}} J(j) \Phi(j) \Delta_{\alpha} j-\int_{\left[\ell_{1}, \ell_{1}+\hat{\Im}_{2}\right]_{\mathbb{T}}} J(j) \Delta_{\alpha}\right]+J\left(\ell_{2}\right)\left(\hat{\Im}_{2}-\int_{\left[\ell_{1}, \ell_{2}\right]_{\mathbb{T}}} \Phi(j) \Delta_{\alpha \jmath}\right) \\
\leq-M \int_{\left[\ell_{1}, \ell_{2}\right]_{\mathbb{T}}}\left|J(\jmath)-f\left(\ell_{1}+\int_{\left[\ell_{1}, \ell_{2}\right]_{\mathbb{T}}} \Phi(j) \Delta_{\alpha}\right)\right| \Delta_{\alpha \jmath},
\end{gathered}
$$

which is the right-hand side inequality in (18).

Similarly, one can show that

$$
\begin{aligned}
& \left.\left.\int_{\left[\ell_{1}, \ell_{2}\right]_{\mathbb{T}}} I(j) \Phi(j) \Delta_{\alpha}-\int_{\left[\ell_{2}-\hat{\Im}_{1}, \ell_{2}\right]_{\mathbb{T}}} I(j) \Delta_{\alpha}\right]+I\left(\ell_{2}\right)\left(\int_{\left[\ell_{1}, \ell_{2}\right]_{\mathbb{T}}} \Phi(j) \Delta_{\alpha}\right]-\hat{\Im}_{2}\right) \\
& \left.\left.\geq \int_{\left[\ell_{1}, \ell_{2}\right]_{\mathbb{T}}}\left[I(j)-J\left(\ell_{2}\right)\right] \Phi(j) \Delta_{\alpha}\right]+\int_{\left[\ell_{2}-\int_{\left[\ell_{1}, \ell_{2}\right]_{\mathbb{T}}} \Phi(j) \Delta_{\alpha}, \ell_{2}\right]}\left[I\left(\ell_{2}\right)-I(j)\right] \Delta_{\alpha}\right] \\
& \geq M \int_{\left[\ell_{1}, \ell_{2}\right]_{\mathbb{T}}}\left|J(j)-f\left(\ell_{2}-\int_{\left[\ell_{1}, \ell_{2}\right]_{\mathbb{T}}} \Phi(j) \Delta_{\alpha}\right)\right| \Delta_{\alpha},
\end{aligned}
$$

which is the left-hand side inequality in (18).

Corollary 6. Putting $\mathbb{T}=\mathbb{R}$ in Theorem 8 , we obtain

$$
\begin{aligned}
& \int_{\left[\ell_{2}-\hat{\Im}_{1}, \ell_{2}\right]} I(\jmath) d_{\alpha \jmath}+I\left(\ell_{2}\right)\left(\int_{\left[\ell_{1}, \ell_{2}\right]} \Phi(j) d_{\alpha \jmath}-\hat{\Im}_{1}\right) \\
& +M \int_{\left[\ell_{1}, \ell_{2}\right]}\left|J(\jmath)-f\left(\ell_{2}-\int_{\left[\ell_{1}, \ell_{2}\right]} \Phi(\jmath) d_{\alpha \jmath}\right)\right| d_{\alpha \jmath} \\
& \leq \int_{\left[\ell_{1}, \ell_{2}\right]} I(j) \Phi(j) d_{\alpha \jmath} \\
& \leq \int_{\left[\ell_{1}, \ell_{1}+\hat{\Im}_{2}\right]} I(j) d_{\alpha \jmath}-J\left(\ell_{2}\right)\left(\hat{\Im}_{2}-\int_{\left[\ell_{1}, \ell_{2}\right]} \Phi(j) d_{\alpha \jmath}\right) \\
& -M \int_{\left[\ell_{1}, \ell_{2}\right]}\left|I(j)-f\left(\ell_{1}+\int_{\left[\ell_{1}, \ell_{2}\right]} \Phi(j) d_{\alpha \jmath}\right)\right| d_{\alpha \jmath},
\end{aligned}
$$

such that

$$
0 \leq \hat{\Im}_{1} \leq \int_{\left[\ell_{1}, \ell_{2}\right]} \Phi(\jmath) d_{\alpha} \leq \hat{\Im}_{2} \leq \ell_{2}-\ell_{1}
$$

Remark 5. [48] [Theorem 3] can be obtained if we put $\alpha=1$ in Corollary 6. 
Theorem 9. If $\Re_{1}, \Re_{2}, \Re_{6}, \Re_{7}$, and $\Re_{14}$ hold, then

$$
\int_{\left[\ell_{1}, \ell_{2}\right]_{\mathbb{T}}} I(\jmath) \Phi(\jmath) \Delta_{\alpha} \leq \int_{\left[\ell_{1}, \ell_{1}+\hat{\Im}\right]_{\mathbb{T}}} I(\jmath) \digamma(\jmath) \Delta_{\alpha} \jmath-\int_{\left[\ell_{1}, \ell_{2}\right]_{\mathbb{T}}}\left|\left(J(\jmath)-I\left(\ell_{1}+\hat{\Im}\right)\right) \psi(\jmath)\right| \Delta_{\alpha} \jmath .
$$

Proof. Follow a similar to the proof of the right-hand side inequality in Theorem 7.

Corollary 7. $\mathbb{T}=\mathbb{R}$ in Theorem, and we get

$$
\left.\int_{\left[\ell_{1}, \ell_{2}\right]} I(\jmath) \Phi(\jmath) d_{\alpha \jmath} \leq \int_{\left[\ell_{1}, \ell_{1}+\hat{\Im}\right]} I(\jmath) \digamma(\jmath) d_{\alpha} \jmath-\int_{\left[\ell_{1}, \ell_{2}\right]}\left|\left(I(\jmath)-I\left(\ell_{1}+\hat{\Im}\right)\right) \psi(\jmath)\right| d_{\alpha}\right] .
$$

Remark 6. If we take $\alpha=1$, in Corollary 7, we recapture [47] [Theorem 2.12].

Corollary 8. Hypotheses $\Re_{1}, \Re_{2}, \Re_{3}, \Re_{10}$, and $\Re_{11}$ yield

$$
\left.\left.\left.\int_{\left[\ell_{1}, \ell_{2}\right]_{\mathbb{T}}} I(j) \Phi(j) \Delta_{\alpha}\right] \leq \int_{\left[\ell_{1}, \ell_{1}+\hat{\Im}\right]_{\mathbb{T}}} I(j) \Delta_{\alpha}\right]-\int_{\left[\ell_{1}, \ell_{2}\right]_{\mathbb{T}}}\left|\left(\frac{I(j)}{\digamma(\jmath)}-\frac{J\left(\ell_{1}+\hat{\Im}\right)}{\digamma\left(\ell_{1}+\hat{\Im}\right)}\right) \digamma(\jmath) \psi(\jmath)\right| \Delta_{\alpha}\right] .
$$

Proof. Insert $\Phi(\jmath) \mapsto \digamma(\jmath) \Phi(\jmath), I(\jmath) \mapsto J(\jmath) / \digamma(\jmath)$ and $\psi(\jmath) \mapsto \digamma(\jmath) \psi(\jmath)$ in Theorem 9 .

Corollary 9. $\mathbb{T}=\mathbb{R}$ in Corollary 8 , we have

$$
\int_{\left[\ell_{1}, \ell_{2}\right]} I(\jmath) \Phi(\jmath) d_{\alpha} \leq \int_{\left[\ell_{1}, \ell_{1}+\hat{\Im}\right]} I(\jmath) d_{\alpha \jmath}-\int_{\left[\ell_{1}, \ell_{2}\right]}\left|\left(\frac{I(\jmath)}{\digamma(\jmath)}-\frac{I\left(\ell_{1}+\hat{\Im}\right)}{\digamma\left(\ell_{1}+\hat{\Im}\right)}\right) \digamma(\jmath) \psi(\jmath)\right| d_{\alpha} \jmath .
$$

Remark 7. [47] [Corollary 2.3] can be recovered with the help of $\alpha=1$, in Corollary 9.

Theorem 10. If $\Re_{1}, \Re_{2}, \Re_{6}, \Re_{7}$, and $\Re_{15}$ hold, then

$$
\left.\int_{\left[\ell_{2}-\hat{\Im}, \ell_{2}\right]_{\mathbb{T}}} I(\jmath) \digamma(\jmath) \Delta_{\alpha} J+\int_{\left[\ell_{1}, \ell_{2}\right]_{\mathbb{T}}}\left|\left(J(\jmath)-J\left(\ell_{2}-\hat{\Im}\right)\right) \psi(\jmath)\right| \Delta_{\alpha} \leq \int_{\left[\ell_{1}, \ell_{2}\right]_{\mathbb{T}}} I(\jmath) \Phi(\jmath) \Delta_{\alpha}\right] .
$$

Proof. Carry out the same proof of the left-hand side inequality in Theorem 7.

Corollary 10. $\mathbb{T}=\mathbb{R}$ in Theorem 10 , and we have

$$
\int_{\left[\ell_{2}-\hat{\Im}, \ell_{2}\right]} I(\jmath) \digamma(\jmath) d_{\alpha \jmath}+\int_{\left[\ell_{1}, \ell_{2}\right]}\left|\left(I(\jmath)-I\left(\ell_{2}-\hat{\Im}\right)\right) \psi(\jmath)\right| d_{\alpha \jmath} \leq \int_{\left[\ell_{1}, \ell_{2}\right]} I(\jmath) \Phi(\jmath) d_{\alpha \jmath} .
$$

Remark 8. If we take $\alpha=1$, in Corollary 10, we recapture [47] [Theorem 2.13].

Corollary 11. Let $\Re_{1}, \Re_{2}, \Re_{3}, \Re_{9}$, and $\Re_{12}$ be fulfilled. Then,

$$
\left.\left.\int_{\left[\ell_{2}-\hat{\Im}, \ell_{2}\right]_{\mathbb{T}}} I(j) \Delta_{\alpha} j+\int_{\left[\ell_{1}, \ell_{2}\right]_{\mathbb{T}}}\left|\left(\frac{I(\jmath)}{\digamma(\jmath)}-\frac{I\left(\ell_{2}-\hat{\Im}\right)}{\digamma\left(\ell_{2}-\hat{\Im}\right)}\right) \digamma(\jmath) \psi(\jmath)\right| \Delta_{\alpha}\right] \leq \int_{\left[\ell_{1}, \ell_{2}\right]_{\mathbb{T}}} I(j) \Phi(j) \Delta_{\alpha}\right] .
$$

Proof. The proof can be completed by taking $\Phi(\jmath) \mapsto \digamma(\jmath) \Phi(\jmath), J(\jmath) \mapsto J(\jmath) / \digamma(\jmath)$, and $\psi(\jmath) \mapsto \digamma(\jmath) \psi(\jmath)$ in Theorem 10.

Corollary 12. $\mathbb{T}=\mathbb{R}$ in Corollary 11 , and we have

$$
\int_{\left[\ell_{2}-\hat{\Im}, \ell_{2}\right]} I(\jmath) d_{\alpha \jmath}+\int_{\left[\ell_{1}, \ell_{2}\right]}\left|\left(\frac{I(\jmath)}{\digamma(\jmath)}-\frac{I\left(\ell_{2}-\hat{\Im}\right)}{\digamma\left(\ell_{2}-\hat{\Im}\right)}\right) \digamma(\jmath) \psi(\jmath)\right| d_{\alpha \jmath} \leq \int_{\left[\ell_{1}, \ell_{2}\right]} I(\jmath) \Phi(\jmath) d_{\alpha \jmath} .
$$

Remark 9. By letting $\alpha=1$, in Corollary 12, we recapture [47] [Corollary 2.4]. 


\section{Conclusions}

In this important work, we discussed some new dynamic inequalities of Steffensentype using delta integral on time scales. By employing the conformable fractional $\alpha$-integral on time scales, several $\alpha$-conformable Steffensen-type inequalities on time scales are proved. Our proposed results show the potential for producing some original continuous, discrete, and quantum inequalities. We further presented some relevant inequalities as special cases: discrete inequalities and integral inequalities. These results may be used to obtain more generalized results of several obtained inequalities before. Symmetry plays an essential role in determining the correct methods to solve dynamic inequalities.

Author Contributions: Conceptualization, resources, and methodology, A.A.E.-D. and H.A.; investigation and supervision, J.A. and H.A.; data curation, A.A.E.-D.; writing-original draft preparation, A.A.E.-D.; writing-review and editing, J.A. and H.A.; project administration, A.A.E.-D. and H.A. All authors read and agreed to the published version of the manuscript.

Funding: This research received no external funding.

Institutional Review Board Statement: Not applicable.

Informed Consent Statement: Not applicable.

Data Availability Statement: Not applicable.

Acknowledgments: This work has been supported by the Polish National Science Centre under the grant OPUS 14 No. 2017/27/B/ST8/01330.

Conflicts of Interest: The authors declare no conflict of interest.

\section{References}

1. Miller, K.S.; Ross, B. An Introduction to the Fractional Calculus and Fractional Differential Equations; Wiley: Hoboken, NJ, USA,1993.

2. Daftardar-Gejji, V.; Jafari, H. Analysis of a system of nonautonomous fractional differential equations involving caputo derivatives. J. Math. Anal. Appl. 2007, 328, 1026-1033. [CrossRef]

3. Kilbas, A.; Srivastava, H.; Trujillo, J. Theory and Applications of Fractional Differential Equations; Elsevier: Amsterdam, The Netherlands, 2006; Volume 204.

4. Podlubny, I. Fractional Differential Equations: An Introduction to Fractional Derivatives, Fractional Differential Equations, to Methods of Their Solution and Some of Their Applications; Elsevier: Amsterdam, The Netherlands, 1998.

5. Khalil, R.; Horani, M.A.; Yousef, A.; Sababheh, M. A new definition of fractional derivative. J. Comput. Appl. Math. 2014, 264, 65-70. [CrossRef]

6. Iyiola, O.S.; Nwaeze, E.R. Some new results on the new conformable fractional calculus with application using d'alambert approach. Progr. Fract. Differ. Appl. 2016, 2, 115-122. [CrossRef]

7. Iyiola, O.S.; Ojo, G.O. On the analytical solution of fornberg-whitham equation with the new fractional derivative. Pramana 2015, 85, 567-575. [CrossRef]

8. Iyiola, O.S.; Tasbozan, O.; Kurt, A.; Çenesiz, Y. On the analytical solutions of the system of conformable time-fractional robertson equations with 1-d diffusion. Chaos Solitons Fractals 2017, 94, 1-7. [CrossRef]

9. Abdeljawad, T. On conformable fractional calculus. J. Comput. Appl. Math. 2015, 279, 57-66. [CrossRef]

10. Benkhettou, N.; Hassani, S.; Torres, D.F. A conformable fractional calculus on arbitrary time scales. J. King Saud Univ.-Sci. 2016, 28, 93-98. [CrossRef]

11. Nwaeze, E.R. A mean value theorem for the conformable fractional calculus on arbitrary time scales. Progr. Fract. Differ. Appl. 2016, 2, 287-291. [CrossRef]

12. Nwaeze, E.R.; Torres, D.F.M. Chain rules and inequalities for the bht fractional calculus on arbitrary timescales. Arab. J. Math 2017, 6, 13-20. [CrossRef]

13. Bohner, M.; Peterson, A. Dynamic Equations on Time Scales: An Introduction with Applications; Birkhäuser Boston, Inc.: Boston, MA, USA, 2001.

14. Bohner, M.; Peterson, A. (Eds.) Advances in Dynamic Equations on Time Scales; Birkhäuser Boston, Inc.: Boston, MA, USA, 2003; MR 1962542.

15. Hilger, S. Analysis on measure chains-A unified approach to continuous and discrete calculus. Results Math. 1990, 18, 18-56. [CrossRef]

16. Fatma, M.K.H.; El-Deeb, A.A.; Abdeldaim, A.; Khan, Z.A. On some generalizations of dynamic Opial-type inequalities on time scales. Adv. Differ. Equ. 2019, 2019, 323.

17. Li, W.N. Some new dynamic inequalities on time scales. J. Math. Anal. Appl. 2006, 319, 802-814. [CrossRef] 
18. Sheng, Q.; Fadag, M.; Henderson, J.; Davis, J.M. An exploration of combined dynamic derivatives on time scales and their applications. Nonlinear Anal. Real World Appl. 2006, 7, 395-413. [CrossRef]

19. Alomari, M. q-Bernoulli inequality. Turk. J. Sci. 2018, 3, 32-39.

20. Ekinci, A. Inequalities for Convex Functions on Time Scales. Twms J. Appl. Eng. Math. 2019, 9, 64-72.

21. Sarikaya, M.Z. New weighted Ostrowski and Chebyshev type inequalities on time scales. Comput. Math. Appl. 2010, 60, 1510-1514. [CrossRef]

22. Rafeeq, S.; Kalsoom, H.; Hussain, S.; Rashid, S.; Chu, Y.M. Delay dynamic double integral inequalities on time scales with applications. Adv. Differ. Equ. 2020, 1, 1-32. [CrossRef]

23. Ghany, H.A.; Hyder, A.A.; Zakarya, M. Exact Solutions of Stochastic Fractional Korteweg de-Vries Equation with Conformable Derivatives. Chin. Phys. B 2020, 29, 030203-030208. [CrossRef]

24. AlNemer, G.; Kenawy, M.R.; Zakarya, M.; Rezk, H.M. Generalizations of Hardy's Type Inequalities via Conformable Calculus. Symmetry 2021, 13, 242. [CrossRef]

25. Agarwal, R.; Bohner, M.; Peterson, A. Inequalities on time scales: A survey. Math. Inequal. Appl. 2001, 4, 535-557. [CrossRef]

26. Agarwal, R.; O’Regan, D.; Saker, S. Dynamic Inequalities on Time Scales; Springer: Cham, Switzerland, 2014.

27. Saker, S.H.; El-Deeb, A.A.; Rezk, H.M.; Agarwal, R.P. On Hilbert's inequality on time scales. Appl. Anal. Discrete Math. 2017, 11, 399-423. [CrossRef]

28. Tian, Y.; El-Deeb, A.A.; Meng, F. Some nonlinear delay Volterra-Fredholm type dynamic integral inequalities on time scales. Discret. Dyn. Nat. Soc. 2018, 8, 5841985. [CrossRef]

29. El-Deeb, A.A.-M.; Bazighifan, O.; Awrejcewicz, J. A Variety of Dynamic Steffensen-Type Inequalities on a General Time Scale. Symmetry 2021, 13, 1738. [CrossRef]

30. El-Deeb, A.A.; Makharesh, S.D.; Baleanu, D. Dynamic Hilbert-Type Inequalities with Fenchel-Legendre Transform. Symmetry 2020, 12, 582. [CrossRef]

31. El-Deeb, A.A.; Bazighifan, O.; Awrejcewicz, J. On Some New Weighted Steffensen-Type Inequalities on Time Scales. Mathematics 2021, 9, 2670. [CrossRef]

32. El-Deeb, A.A.; Baleanu, D. New Weighted Opial-Type Inequalities on Time Scales for Convex Functions. Symmetry 2020, $12,842$. [CrossRef]

33. Anastassiou, G.A. Foundations of nabla fractional calculus on time scales and inequalities. Comput. Math. Appl. 2010, 59, 3750-3762. [CrossRef]

34. Anastassiou, G.A. Principles of delta fractional calculus on time scales and inequalities. Math. Comput. Model. 2010, 52, 556-566. [CrossRef]

35. Anastassiou, G.A. Integral operator inequalities on time scales. Int. J. Difference Equ. 2012, 7, 111-137.

36. Sahir, M. Dynamic inequalities for convex functions harmonized on time scales. J. App. Math. Phys. 2017, 5, 2360-2370. [CrossRef]

37. Pečarić, J.E. Notes on some general inequalities. Publ. Inst. Math. 1982, 32, 131-135.

38. Sakerr, S.H.; Kenawy, M.; AlNemer, G.H.; Zakarya, M. Some fractional dynamic inequalities of hardy's type via conformable calculus. Mathematics 2020, 8, 434. [CrossRef]

39. Zakaryaed, M.; Altanji, M.; AlNemer, G.H.; El-Hamid, A.; Hoda, A.; Cesarano, C.; Rezk, H.M. Fractional reverse coposn's inequalities via conformable calculus on time scales. Symmetry 2021, 13, 542. [CrossRef]

40. Chu, Y.; Khan, M.A.; Ali, T.; Dragomir, S.S. Inequalities for $\alpha$-fractional differentiable functions. J. Inequal. Appl. 2017, 2017, 1-12. [CrossRef]

41. Khan, M.A.; Ali, T.; Dragomir, S.S.; Sarikaya, M.Z. Hermite-Hadamard type inequalities for conformable fractional integrals, Revista de la Real Academia de Ciencias Exactas, Físicas y Naturales. Serie A. Matemáticas 2018, 112, $1033-1048$.

42. Set, E.; Gözpnar, A.; Ekinci, A. Hermite-Hadamard type inequalities via confortable fractional integrals. Acta Math. Univ. Comen. 2017, 86, 309-320.

43. Sarikaya, M.Z.A.; Billisik, C.C. Opial type inequalities for conformable fractional integrals via convexity. Chaos Solitons Fractals 2016, 97, 1-7.

44. Sarikaya, M.; Budak, H. New inequalities of opial type for conformable fractional integrals. Turk. J. Math. 2017, 41, 1164-1173. [CrossRef]

45. Sarikaya, M.; Yaldiz, H.; Budak, H. Steffensen's integral inequality for conformable fractional integrals. Int. J. Anal. Appl. 2017, 15, 23-30.

46. Anderson, D.R. Taylor's formula and integral inequalities for conformable fractional derivatives. In Contributions in Mathematics and Engineering; Springer: Berlin/Heidelberg, Germany, 2016.

47. Josip, P.; Anamarija, P.; Ksenija, S. Mercer and Wu- Srivastava generalisations of Steffensen's inequality. Appl. Math. Comput. 2013, 219, 10548-10558.

48. Wu, S.H.; Srivastava, H.M. Some improvements and generalizations of Steffensen's integral inequality. Appl. Math. Comput. 2007, 192, 422-428. . [CrossRef] 